日

\title{
Wire Placement in the Lisfranc Joint Using a 2D Projection-Based Software Application for Mobile C-Arms: an Experimental Study in 20 Cadaver Specimens
}

\author{
B. Swartman*, D. Frere, W. Wei, M. Schnetzke, S. Grechenig, A. \\ Matityahu, N. Beisemann, H. Keil, J. Franke, P. A. Grützner, S. Y. Vetter ${ }^{1}$ BG \\ Trauma Center Ludwigshafen, Clinic for Trauma Surgery and Orthopeadics, Ludwig-Guttmann- \\ Str. 13, 67071 Ludwigshafen, Germany, benedict.swartman@,bgu-ludwigshafen.de
}

\begin{abstract}
In the surgical treatment of Lisfranc injuries, percutaneous wire transfixation of the tarsometatarsal complex can be challenging due to it's small corridors and complex anatomy. A novel 2D projection-based software application detects Kirschner wires (Kwires) and automatically indicates their intended direction as a colored trajectory. The aim was to investigate whether the software facilitates K-wire placement in the Lisfranc joint for the inexperienced and the experienced surgeon.

In 20 cadaver foot specimens, $5 \mathrm{~K}$-wires were retrogradely placed into the tarsometatarsal complex from D1-D5 by an experienced and an inexperienced surgeon, with and without using the application. The specimens were presented in pairs; surgeons and software use were equally randomized. Number of placement attempts, duration of procedure, fluoroscopy time, and number of individual fluoroscopy images were recorded.

Use of the software by the inexperienced surgeon significantly reduced the number of placement attempts from 14.2 to $8.8(\mathrm{p}=0.008)$. The application also reduced the number of fluoroscopy images from 44.8 to 40.8 ( $\mathrm{p}=0.230$ ). Duration of procedure and fluoroscopy time were not affected significantly. The experienced surgeon needed more placement attempts using the software ( 8.2 vs. 9.2; $\mathrm{p}=0.351)$. Duration of procedure, fluoroscopy time and number of fluoroscopy images increased while using the software, yet not significantly.

During percutaneous K-wire transfixation of the Lisfranc joint, the novel software is a useful tool for the inexperienced surgeon. In our chosen study setting, the experienced surgeon did not benefit from the software.

Due to its independency on fixed reference markers or registration processes, the software contributes to the procedures in orthopedic surgery.
\end{abstract}




\section{Introduction}

$\mathrm{T}$ Orthopedic and trauma surgery relies on image-guidance as an essential part of surgical therapy. Besides its use in joint reconstruction or spinal surgery, navigational support is increasingly being developed in the field of traumatology $(1,2)$. One increasingly established option is the navigation, extending from intraoperative $2 \mathrm{D}$ or $3 \mathrm{D}$ scan-supported navigation, through reference-based methods, to CT-controlled procedures (3). Limiting factor often is the dependency on special equipment in order to reference table, bone or surgical instruments $(4,5)$.

In the surgical treatment of Lisfranc injuries, percutaneous wire transfixation of the tarsometatarsal complex can be challenging due to its small corridors and complex anatomy. A novel 2D projection-based software application detects Kirschner wires (K-wires) and automatically indicates their intended direction as a colored trajectory on the fixed 2D image.

The aim of the present study was to investigate the novel software application under anatomical conditions. We hypothesized that number of placement attempts, operating time, fluoroscopy time and number of fluoroscopy images can be improved using the software application and that the software holds benefits for both, experienced and inexperienced surgeons performing transfixation of the Lisfranc joint. Document Header

The empty space on top of the first page, just before the title, will be reserved for a page header containing the EasyChair logos and the volume information. This header will be added to the article when you submit it to EasyChair.

You cannot control this header placement and for this reason should not try to change vertical spacing on the first page. If by mistake you removed the empty space, you can restore it as follows. Place the cursor on the very first line of your document (that is, the first line of the title), select Format $->$ Paragraph in the Microsoft Word menu and set the spacing before the paragraph to 94pt and after to $15 \mathrm{pt}$.

\section{Materials and Methods}

The novel software ('K-wire', Siemens Health Care, Erlangen, Germany) is based on 2D projections of a commercially available image intensifier. The software recognizes parallel edges, such as the shadow of a K-wire. By extension, a trajectory is superimposed on the obtained 2D image, visualizing the intended direction of the K-wire (Figure 1). The system cannot track the K-wire or the patient, therefore a new image has to be obtained after each change of position.

10 pairs of adult lower leg specimens were used for this study. The a priori random sample calculation was coordinated with the biometrics institute at Heidelberg University. The cadavers were preserved by Thiel's method (6). The method preserves the color and consistency of the tissues, allowing almost full range of movement of the joints after specimen. Patients gave informed consent during their lifetime as part of the donation of anatomical gift statement.

The indication for wire placement was the treatment of a fictitious Lisfranc injury, in which $5 \mathrm{~K}$ wires were to be placed retrogradely in the tarsometatarsal complex in D1-D5. $2.0 \mathrm{~mm} \mathrm{~K}$-wires were inserted under 2D fluoroscopy (Cios move, Siemens, Erlangen, Germany). The wire placements were performed by two surgeons. One experienced surgeon (a consultant in a trauma surgery and orthopedics department with 12 years of professional experience) and one inexperienced surgeon (resident in fifth year of orthopedics and trauma surgery training) inserted 5 wires into 10 cadaver

feet, of which one foot was treated with and the opposite one without the software. The allocations of operation technique to operated side (left vs. right) and of pairs to the surgeons were randomized. 
Wire placement in the Lisfranc joint using a 2D projection-based software ...

B. Swartman et al.

Number of placement attempts, duration of procedure, fluoroscopy time, and number of individual fluoroscopy images were recorded. 


\section{Results}

Ten lower leg specimen were operated by each surgeon, 5 with and 5 without the software. Use of the software by the inexperienced surgeon significantly reduced the number of placement attempts from 14.2 (SD 3.35; Range 9-18) to 8.8 (2.17; Range 6-11; $\mathrm{p}=0.008$ ). The application also reduced the number of fluoroscopy images from 44.8 (SD 8.93; Range 36-58) to 40.8 (SD 7.26; Range 30-50; $\mathrm{p}=0.230$ ). Duration of procedure took 511.4 s (SD 203.01; Range 376-870) without and 511.0 s (SD 136.61; Range 314-641) with the software application ( $\mathrm{p}=0.499)$. Fluoroscopy time was slightly decreased from 42.8 s (SD 29.25; Range 24-94) to $38.6 \mathrm{~s}$ (SD 13.01; Range 27-60) using the software without a significant difference $(\mathrm{p}=0.388)$.

The experienced surgeon needed more placement attempts using the software compared to the conventional technique $(8.2 \pm 1.3$ vs. $9.2 \pm 5.50 ; \mathrm{p}=0.351)$. Duration of procedure $(298.0 \pm 70.67$ vs. $376 \pm 207.60 ; \mathrm{p}=0.224)$, fluoroscopy time $(20.4 \pm 5.94$ vs. $23.6 \pm 7.92 ; \mathrm{p}=0.245)$ and number of fluoroscopy images ( $33.0 \pm 4.64$ vs. $35.8 \pm 9.26$; $\mathrm{p}=0.281)$ increased while using the software, yet not significantly.

\section{Discussion}

We analyzed the applicability of a novel software application for the C-arm. Every novel system generally depends on the ease of its integration into routine procedures. Therefore, logistical prerequisites have to be created and the team has to be properly trained. Purchasing new devices or additional equipment in the field of image-guided surgery is often associated with high costs (7). The software application investigated in this study can be installed on a conventional 2D image intensifier. Handling is intuitive and there is no need for additional attachments or instrument enhancements.

The system described by Kraus et al. $(8,9)$ relies on reference markers, which have to be equipped to the instruments. Kraus' application is limited in the choice of anatomical region, for the intracorporeal area of interest as well as the extracorporeal reference markers have to be displayed on the same image (9). The novel program in our study targets the K-wire, which lays directly aside the area of interest.

Limitations of the current study lay in its low predictive power due to the small number of cases. However, in the case of placement attempts by the inexperienced surgeon, we found a significant difference. Concerning the other parameters, trends can still be identified. These trends, produced by the inexperienced surgeon, are in line with the results of Kraus et al. (8). In contrast to Kraus, the experienced surgeon did not benefit from the image guidance. Reasons might be the low number of trials in order to get practice with the software application and the already preexisting surgical experience without it. 


\section{Figures}

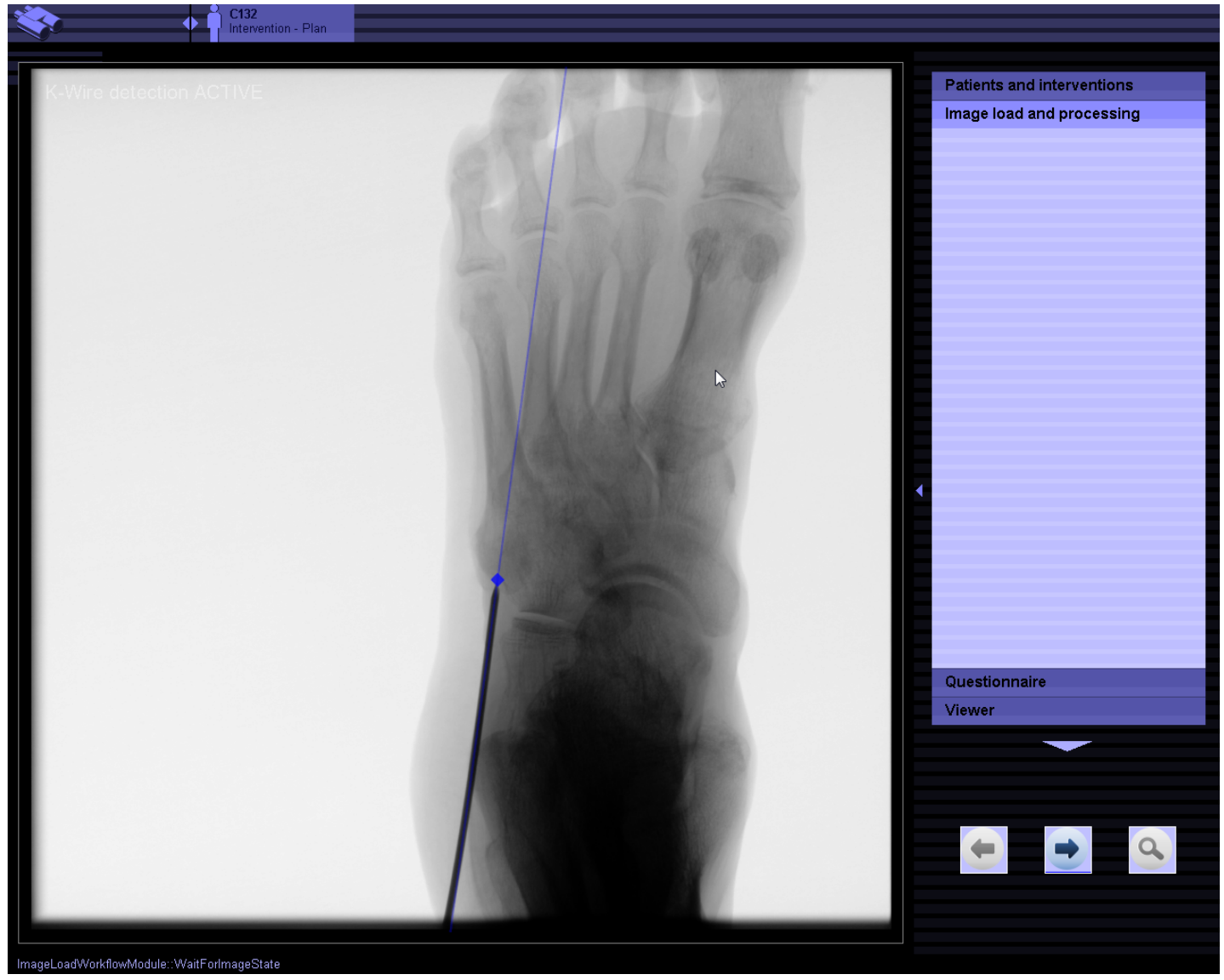

Figure 1: The Screenshot demonstrates the working station for the software application. The program detects the $\mathrm{K}$-wire and visualizes its direction by a superimposed trajectory on the base of an obtained $2 \mathrm{D}$ projection image.

\section{References}

Kahler DM. Image guidance: fluoroscopic navigation. Clinical orthopaedics and related research. 2004(421):70-6. 
Leung KS, Tang N, Cheung LW, Ng E. Image-guided navigation in orthopaedic trauma. The Journal of bone and joint surgery British volume. 2010;92(10):1332-7.

Hufner T, Gebhard F, Grutzner PA, Messmer P, Stockle U, Krettek C. Which navigation when? Injury. 2004;35 Suppl 1:S-a30-4.

Ilsar I, Weil YA, Joskowicz L, Mosheiff R, Liebergall M. Fracture-table-mounted versus bonemounted dynamic reference frame tracking accuracy using computer-assisted orthopaedic surgery--a comparative study. Computer aided surgery : official journal of the International Society for Computer Aided Surgery. 2007;12(2):125-30.

Herman A, Dekel A, Botser IB, Steinberg EL. Computer-assisted surgery for dynamic hip screw, using Surgix, a novel intraoperative guiding system. The international journal of medical robotics + computer assisted surgery : MRCAS. 2009;5(1):45-50.

Thiel W. [The preservation of the whole corpse with natural color]. Annals of anatomy $=$ Anatomischer Anzeiger : official organ of the Anatomische Gesellschaft. 1992;174(3):185-95.

Hartl R, Lam KS, Wang J, Korge A, Kandziora F, Audige L. Worldwide survey on the use of navigation in spine surgery. World neurosurgery. 2013;79(1):162-72.

Kraus M, Weckbach S, Jones A, Krischak G, Gebhard F, Scholl H. Image guidance shortens the learning curve for K-wire placement - an experimental study. The international journal of medical robotics + computer assisted surgery : MRCAS. 2013;9(1):52-7.

Kraus M, von dem Berge S, Scholl H, Krischak G, Gebhard F. Integration of fluoroscopy-based guidance in orthopaedic trauma surgery - a prospective cohort study. Injury. 2013;44(11):1486-92. 